\title{
How Resident Stem Cells Communicate with Cardiac Cells in Beating Heart?
}

\author{
Vincenzo Lionetti ${ }^{1,2,3 *}$ \\ ${ }^{1}$ Laboratory of Medical Science, Institute of Life Sciences, Scuola Superiore Sant'Anna, Pisa, Italy \\ 2Fondazione CNR-Regione Toscana "G. Monasterio", Pisa, Italy \\ ${ }^{3}$ Unit of Translational Medicine, Laboratory of Molecular Biology and Stem Cell Engineering, National Institute of Biostructures and Biosystems, University of Bologna, Italy
}

\begin{abstract}
The adult myocardium is a dynamic tissue with different functions which normally adapts to endless mechanical loads throughout life. The complexity and diversity of myocardial responses to different conditions imply the coexistence of different cell types within a hierarchically ordered architecture. Rare stem/progenitor cells have been detected interspersed within the interstitium and/or adherent to the wall of the capillaries forming the myocardial microcirculation. The origin of these cells is still debated. Multipotent and self-renewing cells resident into the heart survive to a mechanically and biochemically active environment without acquiring a cardiac phenotype. The persistence of an intense physical and biochemical stress do not affect the gene profyle of these cells in beating heart. Otherwise, differentiated cardiac cells continually release humoral factors preserving the fate of stem/progenitor cells. It is conceivable that undifferentiated cardiac cells have a different bio-mechanical response threshold compared to cells resident in other tissue. The codification of the language adopted by cardiac stem/ progenitor cells to communicate with each other and other myocardial cells will help the cardiovascular therapy to fulfill its true potential.
\end{abstract}

\section{Stem cell niche in adult heart}

Numerous evidences showed that the adult heart maintains a weak regenerative potential, such as inherited from the embryonic development, which could contribute to form new muscle after an injury [1-3]. Bergmann et al. [4] reported that cardiomyocytes can renew ( $1 \%$ per year at age $25,0.45 \%$ at age 75 ) by cell cycle reentry to resume mitosis and synthesis DNA. Thus, approximately $50 \%$ of these cells are renewed during a normal lifespan. Several investigators have hypothesized that the plasticity of the adult myocardium depends on the presence of cellular residues of cardiomyogenesis interspersed within the interstitium, which are stem and/or progenitor cells. Stem cells are multipotent, self-renewing and clonigenic cells that can replace apoptotic, dysfunctional and/or senescent cardiomyocytes, fibroblasts and endothelial cells by translineage commitment [5] or cell fusion $[6,7]$. In addition to new insights regarding the presence of endogenous cardiac stem cells, some investigators strongly support the concept that the heart could contain stem cell pools or niches [8], which are mainly located in epicardial myocardium $[9,10]$. During heart development, cells from the proepicardial organ spread over the naked heart tube to form the epicardium. The epicardium-derived cells proved to be indispensable for the formation of the ventricular compact zone and myocardial maturation by interacting directly with cardiomyocytes [11]. In fact the stem/progenitor cells of the niches seem to be connected to the pre-existing cardiomyocytes and interstitial supporting cells [12], but the complete identity of the interstitial cells and the code of the intercellular cross-talk is still unknown. The maintenance of cardiac homeostasis should depend on the survival and activity of stem/ progenitor cells resident into the epicardial niches $[13,14]$. The size of stem cell niche in the adult myocardium might constitutionally vary in each person and might be affected by age [15], comorbidities (ie: high cholesterol, diabetes, dystrophy) [16-19] and the magnitude of cardiac dysfunction [15]. Conversely, Kubo et al. [20] showed that the number of cardiac progenitor cells was significantly increased nearly 4 -fold in patients with end-stage heart failure compared with healthy patients. However, it is still debated whether the circulating stem cells replenish the cardiac niches and guarantee the maintenance of regenerative potential of the adult heart [21-23]. Fortini et al. [24] showed that differently from haematopoietic stem cells and endothelial progenitor cells, the tissue-committed stem cells significantly increased with the progression of the disease, suggesting a possible distinct role of these cells in the pathophysiology of HF. However, the recent experimental findings demonstrating the marked increase of stem/progenitor cells into the failing heart and into the blood stream highlight the notion that the basis of myocardial regeneration is not as important the quality or quantity of resident or engrafted stem/progenitor cells to the myocardium, but how these cells communicate to each other and with cardiac cells functionally mature. In this regard, the role of autocrine and paracrine signals modulating the communication among different resident cells is playing an increasingly important and intriguing point of view for the homeostatic cardiac growth and maintenance [25]. Stem cells and cardiac resident cells interact continuously and dynamically using a physical, biochemical and epigenetic alphabet.

\section{Physical alphabet}

Physical stimuli act on the cell shape and can influence the biochemistry and genotype of resident stem cells in tissue exposed to mechanical forces at different magnitude, such as the beating heart [26]. The focal adhesion complex regulates the traffic of signals from the physical environment to the cellular function [27]. The magnitude of

*Corresponding author: Vincenzo Lionetti, MD, PhD, FAHA,Laboratory of Medical Science,Institute of Life Sciences,Scuola Superiore Sant'Anna,Piazza Martiri della Libertà, 3356124-Pisa, Italy, Tel: +39-050-3152216; Fax: +39-0503152166; E-mail: v.lionetti@sssup.it

Received November 03, 2011; Accepted November 04, 2011; Published November 06, 2011

Citation: Lionetti V (2011) How Resident Stem Cells Communicate with Cardiac Cells in Beating Heart? J Stem Cell Res Ther 1:e104. doi:10.4172/21577633.1000e104

Copyright: (c) 2011 Lionetti V. This is an open-access article distributed under the terms of the Creative Commons Attribution License, which permits unrestricted use, distribution, and reproduction in any medium, provided the original author and source are credited. 
the signals translated by focal adhesion in stem cells is mainly mediated by specific kinases. Hakuno D et al. [28] showed that the kinase of focal adhesion is a key regulator of cardiomyogenesis and can direct specific cardiac commitment of stem cells. Recent findings demonstrated that cells perceive the microenvironment and mediate the cell response trough a novel molecular axis composed by Yorkie-homologues YAP (Yes-associated protein) and TAZ (transcriptional coactivator with PDZ-binding motif, also known as WWTR1), acting as nuclear relays of mechanical signals [29]. This new evidence suggest a new target to modulate stem cell plasticity and activity, which is interfered by the mechanical environment. In this regard, Maioli et al [30] recently observed that radiofrequency waves from Wi-Fi technologies optimize stem cell plasticity and prime cardiac differentiation in undifferentiated cells.

\section{Biochemical alphabet}

To date, a growing body of data suggests that the survival and function of cardiac resident cells are mainly attributed to autocrine and paracrine signaling mechanisms. The release of such humoral biofactors is due to the cells ability to sense cardiac environmentally derived cues, but the exact feedback loops are still poorly understood [25]. The release of various cardiotropic cytokines and growth factors by mesenchymal stem cells underscored the contribution of paracrine mechanisms to activate Notch-1 receptor by its ligand Jagged-1 expressed by the adjacent mesenchymal stem cells [31]. In this regard, the Notch-1 up-regulation enhances the cardiomyocytes proliferation. Moreover, the paracrine factors released by stem cells can increase the survival of cardiac mature cells [32] and angiogenesis [33,34] following ischemic insult. High Mobility Box 1 Protein (HMGB1) is a cytokine released into the extracellular space by necrotic cells and enhances the paracrine response of cardiac fibroblasts by activating resident cardiac c-kit+ cells [35]. The effects triggered by biohumoral mediators have a response threshold modulated by specific receptors, which can vary their density on the dose-dependent basis of feedback action. Auto-/ Paracrine signals might tune the phenotype of cells resident into myocardial niche and ameliorate the cellular interdependence trough the modulation of epigenetic mechanisms, such as DNA methylation, histone modifications, and ATP-dependent chromatin remodeling.

\section{Epigenetic alphabet}

Epigenetic mechanisms are reversible, interdependent, and highly dynamic in condition of chromatin structure and specific gene transcription programs, thereby contributing to the homeostasis of cardiac resident cells. The intercellular cross-talk in myocardial niche is allowed in presence of chromatin sensitive to the microenvironment. Previous study showed that the sustained acetylation of histone type 4 in cardiac resident cells tends to allow tissue homeostasis by increasing cell survival and angiogenic response after the release of growth factors (i.e.: VEGF, HGF) in beating heart; moreover, the scenario due to chronic histone acetylation is characterized by an increased rate of proliferation of cardiac cells specific mechanisms, such as SMAD-7 [36] and should depend on SMAD-7 upregulation [37]. Other study showed that WNT signaling pathway plays important roles in the regulation of histone deacetylase type 1 (Hdac1) during the early stage of cardiomyocyte differentiation and that the downregulation of $\mathrm{Hdac1}$ promotes cardiac differentiation [38]. Epigenetic modifications of histones in cardiac resident cells might be modulated also by physical stimuli, such as laminar shear stress [39]. Recent study showed that caveolin-1, mechanosensitive a protein expressed in lipid rafts of cardiac cells induces cardioprotection trough epigenetic mechanisms. In fact, Cav-1 KO mouse abolished the acetylation of histone (H3 and H4) and increased the methylation of histone in the ischemic preconditioned heart [40]. Additionally, the successful terminal differentiation of stem cells is not dependent on adequate methylation levels, but the methylation needs to be delivered by the maintenance enzyme DNA methyltransferase 1 [41].

\section{Conclusions}

Stem cells resident in beating heart constantly communicate with the extracellular and cellular environment in order to ensure a stable, functional and structural interdependence. The data recently published propose a new paradigm in which the activity of stem cells, in harmony with that of mature cardiac cells, it is more important than the number and typology of resident/engrafted cardiac stem cells to ensure efficient function, even in the presence of myocardial damage. Cardiac stem cells suffer much physical, chemical and epigenetic stimuli, and their sensitivity is based on a receptor-dependent threshold mechanism. The decoding of the alphabet used by cells to communicate each other within the niche of the adult myocardium will enable a better understanding of the role played by rare active cardiac stem cells in healthy myocardium, which significantly increase in failing heart without hampering myocardial remodeling.

\section{Acknowledgements}

This work was supported by grant from the Ministry of Health of Italy (GR2007-683407).

\section{References}

1. Thornburg K, Jonker S, O'Tierney P, Chattergoon N, Louey S, et al. (2011) Regulation of the cardiomyocyte population in the developing heart. Prog Biophys Mol Biol 106: 289-299.

2. Ahuja P, Sdek P, MacLellan WR (2007) Cardiac myocyte cell cycle control in development, disease, and regeneration. Physiol Rev 87: 521-544.

3. Smart N, Bollini S, Dubé KN, Vieira JM, Zhou B, et al. (2011) De novo cardiomyocytes from within the activated adult heart after injury. Nature 474 640-644.

4. Bergmann O, Bhardwaj RD, Bernard S, Zdunek S, Barnabé-Heider F, et al (2009) Evidence for cardiomyocyte renewal in humans. Science 324: 98-102.

5. Bearzi C, Rota M, Hosoda T, Tillmanns J, Nascimbene A, et al. (2007) Human cardiac stem cells. Proc Natl Acad Sci USA 104: 14068-14073.

6. Acquistapace A, Bru T, Lesault PF, Figeac F, Coudert AE, et al. (2011) Human mesenchymal stem cells reprogram adult cardiomyocytes toward a progenitorlike state through partial cell fusion and mitochondria transfer. Stem Cells 29 812-824.

7. Avitabile D, Crespi A, Brioschi C, Parente V, Toietta G, et al. (2011) Human cord blood CD34+ progenitor cells acquire functional cardiac properties through a cell fusion process. Am J Physiol Heart Circ Physiol 300: H1875-H1884.

8. Urbanek K, Cesselli D, Rota M, Nascimbene A, De Angelis A, et al. (2006) Stem cell niches in the adult mouse heart. Proc Natl Acad Sci U S A 103: 92269231.

9. Limana F, Capogrossi MC, Germani A (2011) The epicardium in cardiac repair from the stem cell view. Pharmacol Ther 129: 82-96.

10. Gherghiceanu M, Popescu LM (2010) Cardiomyocyte precursors and telocytes in epicardial stem cell niche: electron microscope images. J Cell Mol Med 14 871-877.

11. Weeke-Klimp A, Bax NA, Bellu AR, Winter EM, Vrolijk J, et al. (2010) Epicardium-derived cells enhance proliferation, cellular maturation and alignment of cardiomyocytes. J Mol Cell Cardiol 49: 606-616. 
12. Dellatore SM, Garcia AS, Miller WM (2008) Mimicking stem cell niches to increase stem cell expansion. Curr Opin Biotechnol 19: 534-540.

13. Wills AA, Holdway JE, Major RJ, Poss KD. (2008) Regulated addition of new myocardial and epicardial cells fosters homeostatic cardiac growth and maintenance in adult zebrafish. Development 135: 183-192.

14. Zhou B, Ma Q, Rajagopal S, Wu SM, Domian I, et al. (2008) Epicardial progenitors contribute to the cardiomyocyte lineage in the developing heart. Nature 454: 109-113

15. Cesselli D, Beltrami AP, D’Aurizio F, Marcon P, Bergamin N, et al. (2011) Effects of age and heart failure on human cardiac stem cell function. Am J Pathol 179: 349-366.

16. Chu L, Hao H, Luo M, Huang Y, Chen Z, et al. (2011) Ox-LDL modifies the behaviour of bone marrow stem cells and impairs their endothelial differentiation via inhibition of Akt phosphorylation. J Cell Mol Med 15: 423-432.

17. Cramer C, Freisinger E, Jones RK, Slakey DP, Dupin CL, et al. (2010) Persistent high glucose concentrations alter the regenerative potential of mesenchymal stem cells.Stem Cells Dev 19: 1875-1884.

18. Rota M, LeCapitaine N, Hosoda T, Boni A, De Angelis A, et al. (2006) Diabetes promotes cardiac stem cell aging and heart failure, which are prevented by deletion of the p66shc gene. Circ Res 99: 42-52.

19. Hsiao LC, Carr C, Clarke K (2011) Characterisations of heart function and cardiac stem cells in the animal model of human muscular dystrophy: $\mathrm{mdx}$ mice. Heart 97: e7.

20. Kubo H, Jaleel N, Kumarapeli A, Berretta RM, Bratinov G, et al. (2008) Increased cardiac myocyte progenitors in failing human hearts. Circulation 118 : 649-657.

21. Bittner RE, Schöfer C, Weipoltshammer K, Ivanova S, Streubel B, et al. (1999) Recruitment of bone-marrow-derived cells by skeletal and cardiac muscle in adult dystrophic mdx mice. Anat Embryol (Berl) 199: 391-396.

22. Castellani C, Padalino M, China P, Fedrigo M, Frescura C, et al. (2010) Bonemarrow-derived CXCR4-positive tissue-committed stem cell recruitment in human right ventricular remodeling. Hum Pathol 41: 1566-1576.

23. Visconti RP, Markwald RR (2006) Recruitment of new cells into the postnata heart: potential modification of phenotype by periostin. Ann N Y Acad Sci 1080: 19-33.

24. Fortini C, Toffoletto B, Fucili A, Puppato E, Olivares A, et al. (2011) Circulating stem cell vary with NYHA stage in heart failure patients. J Cell Mol Med 15 1726-1736.

25. Lionetti V, Bianchi G, Recchia FA, Ventura C (2010) Control of autocrine and paracrine myocardial signals: an emerging therapeutic strategy in heart failure Heart Fail Rev 15: 531-542

26. Lionetti V, Cecchini M, Ventura C (2011) Nanomechanics to drive stem cells in injured tissues: insights from current research and future perspectives. Stem Cells Dev 20: 561-568

27. Song H, Cha MJ, Song BW, Kim IK, Chang W, et al. (2010) Reactive oxygen species inhibit adhesion of mesenchymal stem cells implanted into ischemic myocardium via interference of focal adhesion complex. Stem Cells 28: 555563

28. Hakuno D, Takahashi T, Lammerding J, Lee RT (2005) Focal adhesion kinase signaling regulates cardiogenesis of embryonic stem cells. J Biol Chem 280: 39534-39544.

29. Dupont S, Morsut L, Aragona M, Enzo E, Giulitti S, et al. (2011) Role of YAP/ TAZ in mechanotransduction. Nature 474: 179-183.

30. Maioli M, Rinaldi S, Santaniello S, Castagna A, Pigliaru G, et al. (2011) Radio Frequency Energy Loop Primes Cardiac, Neuronal, and Skeletal Muscle Differentiation in Mouse Embryonic Stem Cells: A New Tool For Improving Tissue Regeneration. Cell Transplant doi: 10.3727/096368911X600966.

31. Sassoli C, Pini A, Mazzanti B, Quercioli F, Nistri S, et al. (2011) Mesenchymal stromal cells affect cardiomyocyte growth through juxtacrine Notch-1/Jagged-1 signaling and paracrine mechanisms: clues for cardiac regeneration. J Mol Cell Cardiol 51: 399-408
32. Angoulvant $D$, Ivanes $F$, Ferrera $R$, Matthews PG, Nataf $S$, et al. (2011) Mesenchymal stem cell conditioned media attenuates in vitro and ex vivo myocardial reperfusion injury. J Heart Lung Transplant 30: 95-102.

33. Li H, Zuo S, He Z, Yang Y, Pasha Z, et al. (2010) Paracrine factors released by GATA-4 overexpressed mesenchymal stem cells increase angiogenesis and cell survival. Am J Physiol Heart Circ Physiol 299: H1772-H1781.

34. Timmers L, Lim SK, Hoefer IE, Arslan F, Lai RC, et al. (2011) Human mesenchymal stem cell-conditioned medium improves cardiac function following myocardial infarction. Stem Cell Res 6: 206-214.

35. Rossini A, Zacheo A, Mocini D, Totta P, Facchiano A, et al. (2008) HMGB1 stimulated human primary cardiac fibroblasts exert a paracrine action on human and murine cardiac stem cells. J Mol Cell Cardiol 44: 683-693.

36. Lionetti V, Cantoni S, Cavallini C, Bianchi F, Valente S, et al. (2010) Hyaluronan mixed esters of butyric and retinoic acid affording myocardial survival and repair without stem cell transplantation. J Biol Chem 285: 9949-9961.

37. Maioli M, Santaniello S, Montella A, Bandiera P, Cantoni S, et al. (2010) Hyaluronan esters drive Smad gene expression and signaling enhancing cardiogenesis in mouse embryonic and human mesenchymal stem cells. PLoS One 5: e15151.

38. Liu Z, Li T, Liu Y, Jia Z, Li Y, et al. (2009) WNT signaling promotes Nkx2.5 expression and early cardiomyogenesis via downregulation of Hdac1. Biochim Biophys Acta 1793: 300-311.

39. Illi B, Scopece A, Nanni S, Farsetti A, Morgante L, et al. (2005) Epigenetic histone modification and cardiovascular lineage programming in mouse embryonic stem cells exposed to laminar shear stress. Circ Res 96: 501-508.

40. Das M, Das S, Lekli I, Das DK (2011) Caveolin induces cardioprotection through epigenetic regulation. J Cell Mol Med doi: 10.1111/j.1582-4934.2011.01372.x

41. Jackson M, Krassowska A, Gilbert N, Chevassut T, Forrester L, et al. (2004) Severe global DNA hypomethylation blocks differentiation and induces histone hyperacetylation in embryonic stem cells. Mol Cell Biol 24: 8862-8871. 